\title{
HAADF STEM Observations of Impurity Distributions around Basal Dislocation in Alumina
}

\author{
Eita Tochigi ${ }^{1}$, Yuki Kezuka ${ }^{1}$, Akiho Nakamura ${ }^{1}$, Atsutomo Nakamura ${ }^{2}$, Naoya Shibata ${ }^{1}$, and Yuichi \\ Ikuhara $^{3,4}$
}

1. Institute of Engineering Innovation, The University of Tokyo, Tokyo, Japan

2. Department of Materials Science and Engineering, Nagoya University, Nagoya, Japan

3. Nanostructures Research Laboratory, Japan

Fine Ceramics Center, Nagoya, Japan

4. WPI-AIMR Research Center, Tohoku University, Sendai, Japan

Dislocations strongly interact with impurities in a crystal and form impurity atmospheres along their line directions. Impurity distributions around a dislocation have been mainly explained by an elastic interaction. Furthermore, it is presumably considered that an electrostatic interaction between dislocations and impurities also affects to impurity distributions, especially in ionic crystals. However, the electrostatic interaction has not been well understood yet. It has been reported that the $1 / 3<11-20>$ edge basal dislocation in alumina $\left(\alpha-\mathrm{Al}_{2} \mathrm{O}_{3}\right)$ is dissociated into two partial dislocations with nonstoichiometric cores [1]. Thus, a strong electrostatic interaction is expected to act between the partial dislocation cores and impurities. In this study, we observed distributions of different impurities around the dissociated basal dislocations of alumina at the atomic scale to investigate the electrostatic interaction.

We fabricated alumina bicrystals including a $\{11-20\}<1-100>2^{\circ}$ low-angle tilt grain boundary with and without impurities, because alumina $\{11-20\}<1-100>$ low-angle tilt grain boundaries without impurities are known to consist of the dissociated basal edge dislocations [2]. The impurity-doped bicrystals were obtained to join pairs of a single crystal with a metal-deposited surface by diffusion bonding as shown in Fig. 1a. The resultant grain boundaries were observed by high-angle annular dark-field scanning transmission electron microscopy (HAADF STEM: JEOL JEM-2100F with a CEOS Cs-corrector operated at 200kV). Fig. 2a shows a HAADF STEM image of a $1 / 3<11-20>$ basal dislocation without impurities. The positions of the dislocation cores are indicated by the arrows. It is found that the basal dislocation is dissociated into $1 / 3<10-10>$ partial dislocations. We found this type of dissociated structure in all the impurity-doped boundaries we fabricated. Fig. $2 \mathrm{~b}$ shows a strain map of $\varepsilon_{\mathrm{xx}}$ of a basal dislocation in the $\{11-20\}<1-100>2^{\circ}$ low-angle tilt grain boundary theoretically calculated by the Pielers-Nabbaro model $[3,4]$. The lower partial has the lager tensile strain field than the upper partial because of the characteristic configuration of dislocations in the grain boundary. Thus, the lower partial will have a larger impurity atmosphere if an elastic strain effect is predominant for impurity distribution. Presumably, distributions of $\mathrm{Er}^{3+}$ agreed well to the strain fields, because the $\mathrm{Er}^{3+}$ ion is considered to be substituted for $\mathrm{Al}^{3+}$ ions with charge neutrality. However, distributions of ions having $2+$ or $4+$ valency did not agree to the strain fields. This may be because these ions distributed to compensate the charge of the nonstoichiometric cores rather than the size mismatch. In the presentation, we will discuss on the relationship between the observed impurity distributions and the dislocation core structures on the basis of the ion size or the valency of the impurities. 


\section{References:}

[1] N. Shibata et al, Science 316 (2007) p. 82.

[2] A. Nakamura et al, Philos. Mag. 86 (2006) p. 4657.

[3] R. Peierls, Proc. Phys. Soc. London. 52 (1940) p. 34.

[4] F. R. N. Nabarro, Proc. Phys. Soc. London. 59 (1947) p. 34.

(a)

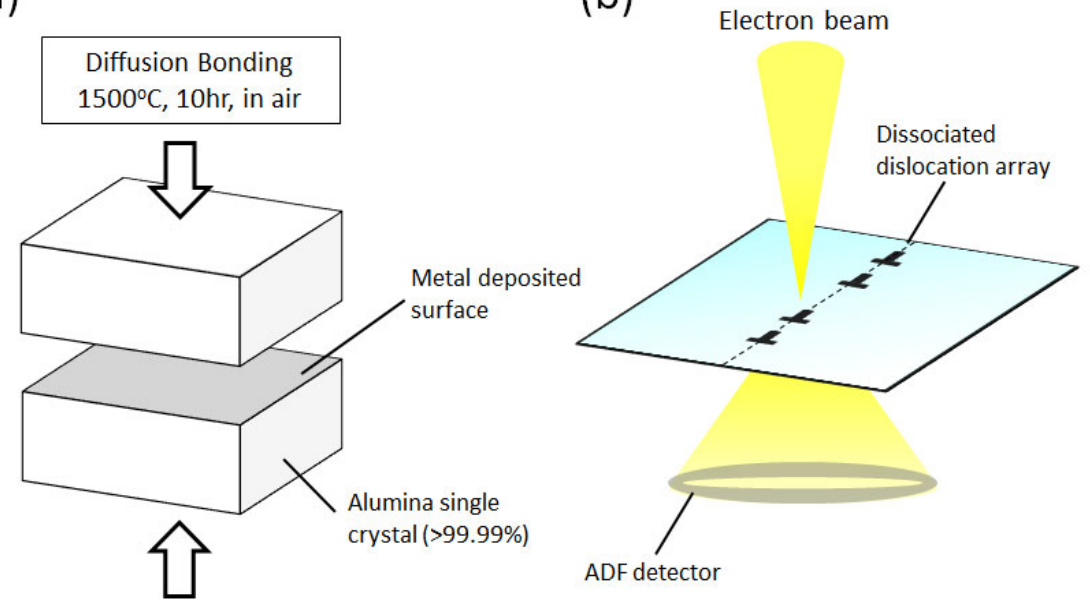

Figure 1. (a) A schematic illustration of fabrication of an impurity-doped bicrystal. (b) A schematic illustration showing ADF STEM analysis of a low-angle tilt boundary in alumina.
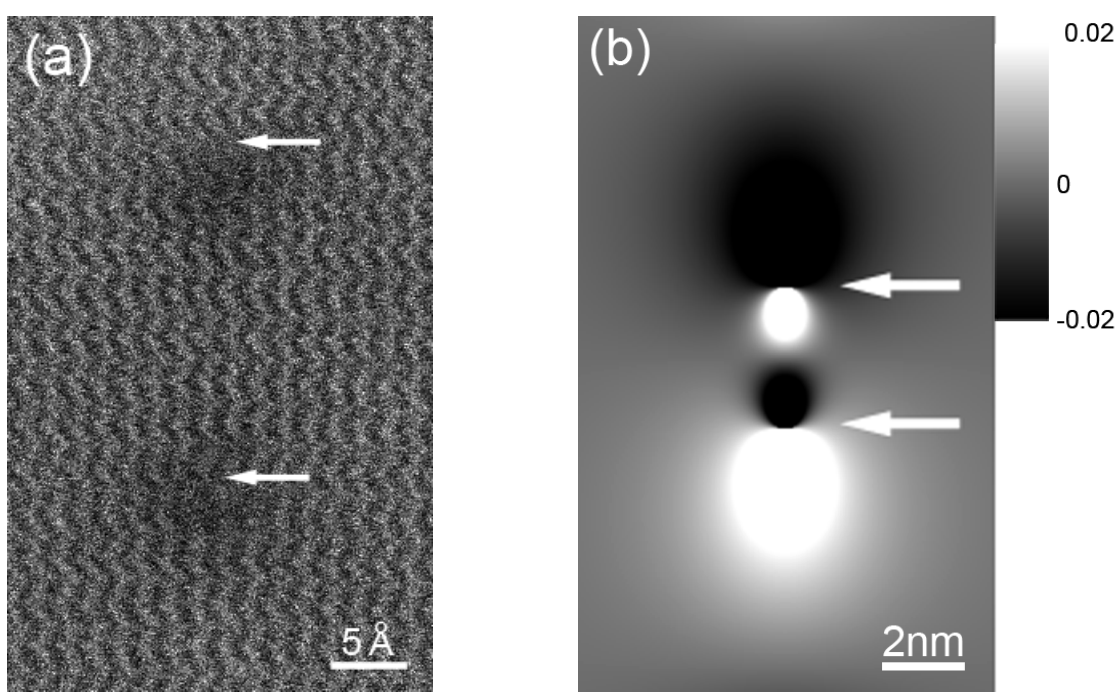

Figure 2. (a) A HAADF STEM image of a $1 / 3<11-20>$ basal dislocation without impurity. (b) A calculated map of strain $\boldsymbol{\varepsilon}_{\mathrm{xx}}$ of a basal dislocation in the $\{11-20\}<1-100>2^{\circ}$ low-angle boundary. 